\title{
Biobanking for the future, how to prepare for the next generation of Next Generation Sequencing
}

\section{Tomas Klingström}

Swedish University of Agricultural Sciences, Uppsala, Sweden Klingström T (2015) EMBnet.journal 21(Suppl A), e815. ntttp://dx.dọ.org/10.14806/ej.21.A. . 815

In biobanking the quality of samples is defined depending on their usage. A piece of formalinfixed, paraffin-embedded tissue may therefore be considered of very high quality when used for immune histochemistry, despite heavy crosslinking and fragmentation of nucleic acids. This, combined with ethical and legal questions on informed consent, makes it important for biobanks to prepare their collections for future technology.

The technology watch of the BBMRI-LPC 2015 will focus on the pre-analytical, ethical and data management issues that may prevent biobanks from providing samples that fully take advantage of the next generation of sequencing technology. Biobanks are a long term commitment, but by preparing for the next generation of technology, biobanks provide researchers with immediate access to material for testing scientific theories that would otherwise take years or decades to evaluate.
To properly support next generation sequencing it is therefore especially important that biobanks consider the following challenges:

- technical challenges - DNA is often regarded as stable and easy to work with. But NGS with long read lengths, epigenetic studies and single cell sequencing are all areas where current or upcoming technologies will greatly challenge the quality management of biobanks;

- ethical challenges - every sample may be a genetic sample if the research is innovative and meticulous enough. This greatly increases the requirements on how biobanks handle donor consent and privacy when collecting biospecimen;

- data management - despite huge cost reduction, NGS remains the costliest step in the process. Data access to ensure reuse and repeatability is therefore important. 